\title{
Assessing the strength of angular induction using waveforms
}

\author{
ERNEST GREENE \\ University of Southern California, Los Angeles, California \\ and \\ SABAH AL-QUADDOOMI \\ Kuwait University, Safat, Kuwait
}

\begin{abstract}
Angular induction is the perceptual process by which one line can bias the perceived orientation of another line or its projection. These effects are generally evaluated using long, straight lines, but the use of waveforms provides a way to vary the orientation of adjacent segments systematically. This may provide information about the nature of local and global interactions.
\end{abstract}

The biological and perceptual mechanisms for judging the straightness and alignment of lines and points over long distances are not well understood. However, it is known that these attributes are affected by the relative position of the elements, as seen in classic illusions of angle and direction. For example, an oblique line segment will be misprojected across the space that lies between two horizontal or vertical parallels; this is one of the standard variants of the Poggendorff illusion. The direction of the misprojection is similar to the bending of light as it passes from air into water, but the degree of misprojection is a function of the angle of the oblique relative to each of the induction lines (Greene, 1987, 1988). Essentially, horizontal and vertical lines have opposite effects on projection of the oblique, thus the influence of one line virtually cancels the effect of the other, even though they lie at different distances along the path of the projection.

A long horizontal or vertical line segment has a greater influence on the projection than does a short segment. The present research examines the question of whether long lines derive their influence by the simple linear combination of effects from component segments. In this study, several patterns of line elements were constructed in a way that provided differential "loading" of components (e.g., a square waveform composed of successive short vertical and horizontal segments). Our goal was to evaluate the overall influence of the combined elements for inducing misprojection.

\section{METHOD}

Visual stimulus materials were created using an Apple Macintosh computer and a laser printer (Adobe Illustrator software). Sinusoid, square, and triangular waveforms were presented on the page as parallel bands,

This work was supported in part by the Neuropsychology Foundation. Sabah Al-Quaddoomi was on a sabbatical leave at the University of Southern California from Kuwait University for the 1987-88 academic year. Correspondence may be addressed to Ernest Greene, Department of Psychology, University of Southern California, Los Angeles, CA 90089. as illustrated in Figure 1. Each page also contained an oblique-line segment in one of the quadrants, positioned at $30^{\circ}$ to the horizontal. The general configuration was the same as for one diagonal of a Poggendorff illusion.

Each of the parallel waveforms consisted of a repeating cycle of a particular amplitude and wavelength of sine, square, left-facing, and rightfacing triangular (sawtooth) waves. The amplitude and wavelength of a 1-unit wave was sized to be seen as $20 \mathrm{~min}$ of visual angle when viewed at $46 \mathrm{~cm}$. The sizing was designed to allow comparison to a biological model, as illustrated in Figure 2 and discussed below. The 2-unit wave maintained the same amplitude, but completed one cycle in $\mathbf{4 0}$ min of visual angle. By this method of "stretching" the waveform, a set was constructed that contained 1-, 2-, 4-, and 8-unit wavelengths for each of the wave shapes, as shown in Figure 1. In addition, the test material contained pages in which simple horizontal lines were used instead of the waveforms. This condition provided a comparison standard for expressing the waveform effects as "percent induction."

The influence of the waveforms in causing misprojection of an oblique was evaluated with two sets of subjects under somewhat different test conditions. For one set of subjects (the large-group condition), the oblique was presented in the upper right or upper left quadrant of the page, and the subjects were asked to 'mark the lower 'target wave' at the spot where the oblique would strike if it were drawn across the space between the two waves." Fifty-five undergraduates at the University of Southern California were tested in this group. The subjects were seated together in a classroom. Each was provided with a booklet of clean, plain-paper copies of the stimulus material that used one of four random orders of wave shape, length, and oblique position $(4 \times 4 \times 2)$, plus the two comparison standards with horizontal lines rather than waveforms (for a total of 34 stimulus configurations). The booklet also contained a diagram of task requirements and written instructions, including requests that they keep the pages oriented horizontally and avoid twisting their head as they judged the projection.

In the small-group condition, 5 subjects were tested individually using a viewing stand and mask, following the methods of Weintraub \& Brown (1986). The stand provided a viewing/marking surface inclined at $15^{\circ}$, with edge barriers to aid in the alignment of the stimulus pages. It also positioned a mask with eye slots held $46 \mathrm{~cm}$ above the viewing surface. The horizontal alignment of the eye slots discouraged head turning, and thus promoted consistent alignment of the head and eyes as the stimulus pages were being judged.

Obliques were presented in lower as well as upper quadrants, which required that projection be done in each of four directions, and thus increased the number of configurations in the packet to 68 . In addition, each subject was tested twice, with a different random order of the 68 configurations provided in each test session. The average of these two sessions provided the summary data for each subject. 


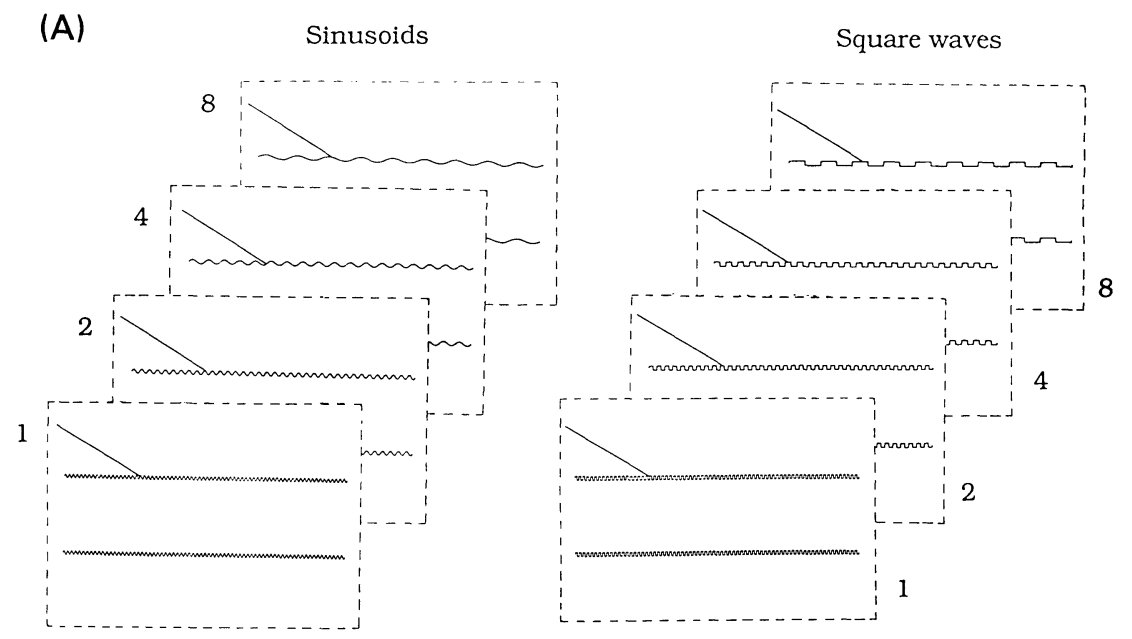

(B) Left-facing Triangular

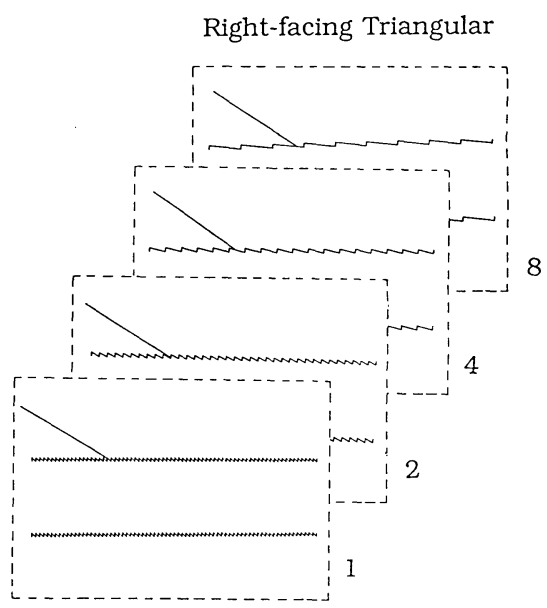

Figure 1. (A) Sinusoid and square waves as they appeared on the $81 / 2 \times 11$ in. page. Each waveform was $23 \mathrm{~cm}$ long, and the two waves were separated by $6 \mathrm{~cm}$ (measured to the central axes). The obliques were $8 \mathrm{~cm}$ long and were placed at $30^{\circ}$ relative to the horizontal. The figure shows the oblique in the upper left corner, but the stimulus package contained figures with the oblique in all four quadrants. The true projection was through the center of the page for each stimulus configuration. (B) Left-facing and right-facing triangular waveforms.

\section{RESULTS}

Misprojection was defined as the angle of departure from the true projection of the oblique. For each subject, the size of the misprojection was calculated as a percentage of that subject's response to the horizontal-line standard; thus, for each task condition the misprojection is expressed as percent induction. The scores of the large group were evaluated with a two-way analysis of variance, and the mean for each task condition is shown in Figure 3. The shape of the wave as well as the wavelength were significant influences, and there was a significant interaction between these task conditions. It is apparent from inspection of the figure that the principal source of difference among the waveforms was the relative effectiveness of the triangular waves in causing misprojection of the obliques. At the longest wavelength, the inductions that resulted from the triangular waves were essentially equivalent to those of the straight-line standard (i.e., $100 \%$ induction strength).

Figure 4 shows the results from intensive testing of the small group of subjects. The same general pattern of effects can be seen in the data of the small group, as well as in the responses of individual subjects (shown in the inset). The effects for the square and sinusoid waves were roughly the same as those for the large group. The induction by the sinusoid wave appeared to increase somewhat with increasing wavelength, but the square-wave induction did not increase as its wavelength increased. Again, the triangular waves benefited the most from an increase in the length of their component segments. For several of the subjects, the effects of the longest triangular waves were above the $100 \%$ level, which suggests that the misprojection influence of long horizontal lines does not provide an upper limit for induction strength. 


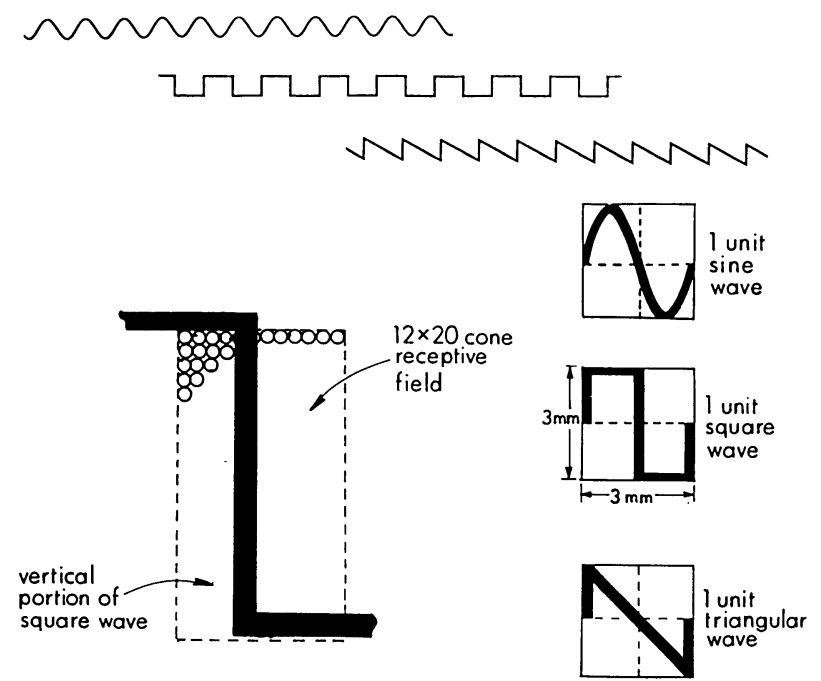

Figure 2. Schematic diagram of the amplitude and length of each wave cycle. These wavelengths were selected so that the results could be interpreted in relation to the model of Dow et al. (1984), who found that orientation-sensitive cells have a $12 \times 20$ cone receptive field.

In an effort to determine why the triangular waves had more influence than the other waveforms, the small-group data were reexamined. The size of the misprojection was greater for those stimulus pages in which the oblique struck the face of the wave at a high incidence (i.e., an acute angle), and smaller where the encounter was at a low incidence. On the basis of this observation, the data were retabulated, combining responses to right- and leftfacing waves but separating the data according to the angle of encounter. Mean values for this summary are shown in Figure 5. It is apparent that misprojection was greater when the incidence of encounter was at a high angle; at long wavelengths the effects were one-third greater than the misprojection that was induced by standard horizontal lines. Nonetheless, both low- and high-incidence stimuli showed a similar increase in induction strength as wavelength increased. This result is also seen in the prior data from the sinusoid.

\section{DISCUSSION}

Long horizontal lines induce misprojection of an oblique in one direction, and long vertical lines bias the projection the other way. The present experiment was designed to test the interaction among vertical and horizontal components at the local level, and to provide information about how local influences are combined over a larger scale.

We chose the size of the basic waveform to correspond to the optimal size of the receptive field of orientation-sensitive neurons, as reported by Dow, Bauer, Snyder, and Vautin (1984). These investigators found that the size of the receptive field in the striate cortex of macaque monkeys is consistent across a large extent of the visual field. They estimated the optimal receptive field size to be about $12 \times 20$ cones, which would mean that the overlap of all fields serving a hypercolumn would be about 20 cones in diameter. We sized the basic wave components according to this estimate, expecting to find a systematic influence of different wavelengths and shapes that might be interpreted as a combination of interactions in a rigid mosaic lattice.

In particular, we expected that the degree of induction would be related to the relative dominance of horizontal-line components in each wave (i.e., where the average tangent vector of the wave approximated the horizontal). In this view, the short-wavelength square waves should not have resulted in a high degree of induction because of mutual cancellation of the effects of the horizontal- and vertical-line components. However, "stretching" the wave (increasing the wavelength) should have increased the ratio of horizontal-to-vertical elements, and would therefore be expected to increase the induction strength of the waveform.

Contrary to these expectations, increasing the wavelength of the squarewave stimulus did not result in greater misprojection of the oblique, and all three wave shapes resulted in misprojection even at the shortest wavelength. It appears that the sinusoid and triangular waves followed our prediction, in that increasing their wavelengths (causing the average tangent vector to approach the horizontal) resulted in greater induction strength. However, it is counterintuitive for this to occur when

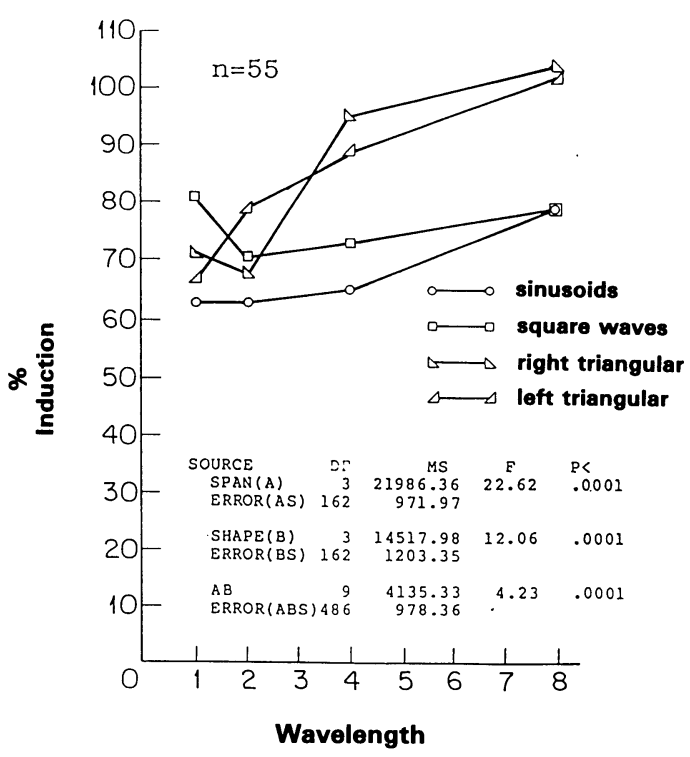

Figure 3. Mean responses of the large subject group showing the effectiveness of each waveform for inducing misprojection of the oblique. Analysis of variance indicated significant differences for the various shapes and wavelengths, as well as an interaction between these two factors.

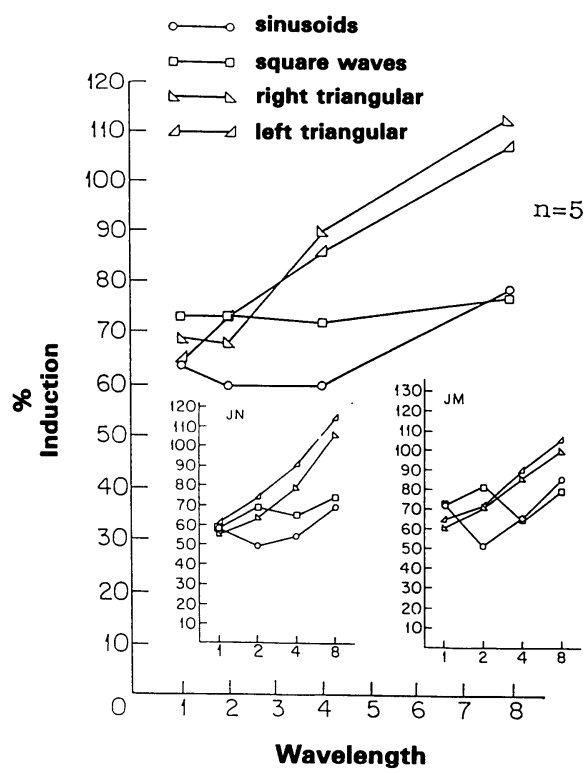

Figure 4. Mean responses of the small subject group, which show a pattern similar to that of Figure 3. The responses of individual subjects are shown in the insets. 


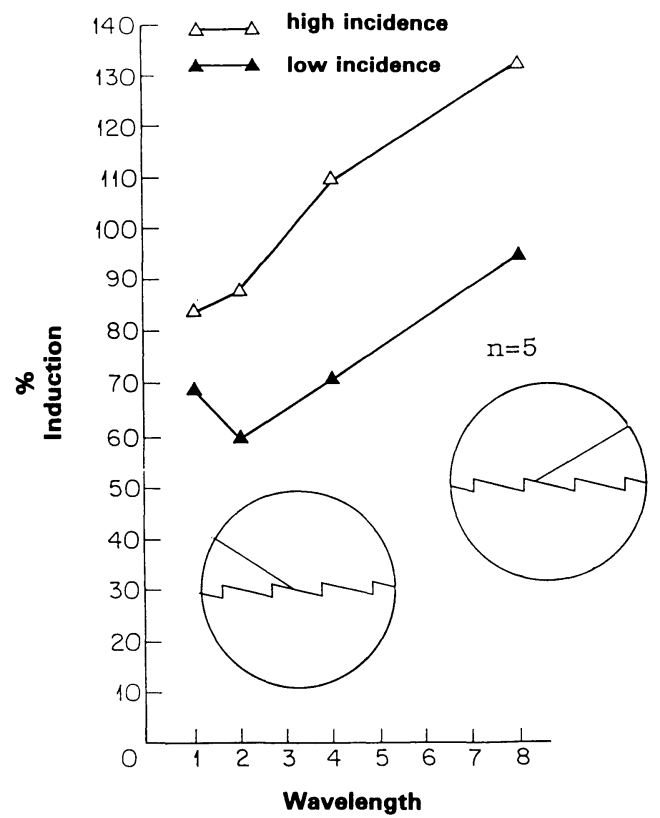

Figure 5. Mean responses of the small subject group to the triangular-waveform stimulus. The induction strength of the waves is affected by the angle at which the oblique strikes the face of the wave. However, it is not clear why the misprojection created by long triangular waves is one-third larger than that produced by horizontal lines.

the square wave itself derived no benefit from increasing the length of its horizontal segments.

The results shown in Figure 5 raise the possibility that peak induction influence may be determined by the size of the angle formed by the oblique and the induction segments. Our experience with manipulation of this angle (Greene \& Al-Quaddoomi, 1989) does show the strength of induction to be a function of this angle. However, in keeping with the experience of
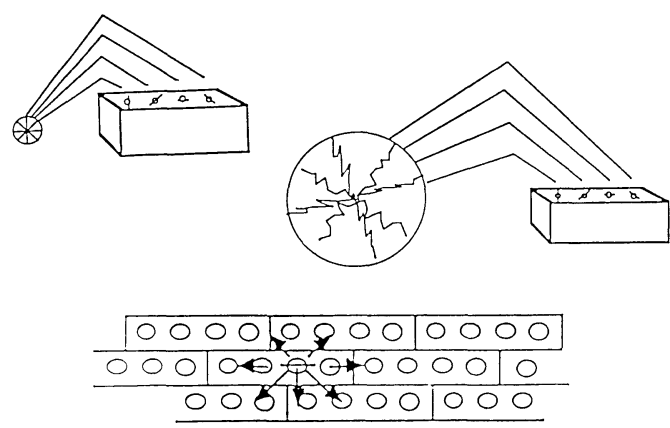

Figure 6. A model summarizing the orientation of stimulus materials at two or more levels of scale. Induction is thought to be the lateral influence within and among the hypercolumns, providing a bias of the perceived orientation of other lines and/or their projections. many investigators who have used long-line stimuli, we have found that the horizontal segments are especially effective. Thus, it is unclear how the triangular configuration is able to achieve such high levels of induction.

There appears to be a $60 \%-80 \%$ "baseline" of induction that is present for all waveforms, upon which other aspects of shape and length are able to build. We are drawn to the view that a substantial portion of the induction is determined by the existence of the two horizontal "bands" of material that stretch across the page as parallels. On a large scale, each is essentially equivalent to what would be classified as a horizontal-line segment were it reduced in scale for presentation to local orientationsensitive fields. Perhaps the molar system provides information about relative alignment among points and segments, leading to a decision about the overall orientation of material across a broad zone. The biological substrates for such a model might be similar to the local system, as suggested in Figure 6. We hypothesize that orientation sensitivity is evaluated on at least two levels, with dedicated hypercolumns responding to the orientation of material that lies in the receptive field, and the only difference is the scale of the responsive zone. Induction would thus be viewed as a lateral bias of responding in the hypercolumn pool, as suggested by Bouma and Andriessen (1970). Overall induction might be a simple combination (e.g., summing) of the local and global effects.

According to this model, any and all waveforms would have a certain potential for induction by dint of the broad, horizontal sweep of the running wave. Thus, at the molar level, the particular shape of the wave, its continuity, and similar factors would be expected to be irrelevant. However, at the local level, the various wave shapes and lengths would have differential effects on projection, and these local influences would be combined with the global effects to determine the overall strength of induction.

Dow et al. (1984) have argued that the size of the optimal receptive field is consistent across a large portion of the primary visual cortex. Our model calls for at least two sizes of receptive field, though we have no reason to insist that both are present at the same brain site. Moreover, it should be noted that Dow et al. have suggested the single-size mosaic on the basis of the relatively flat slope of the average measure of field size as a function of eccentricity. As to the specific sizes that were observed, they showed variation of about five orders of magnitude, which provides some latitude for a model such as ours. Furthermore, as the size of the receptive field grows very large, the number of hypercolumns that are needed to map the entire visual field becomes quite small. Therefore, it is possible that there are small pockets of "global hypercolumns" that might be missed in the sampling process.

\section{REFERENCES}

Bouma, H., \& ANDRIESSEN, J. (1970). Induced changes in the perceived orientation of line segments. Vision Research, 10, 333-347.

Dow, B., BAUER, R., SNYDER, A., \& VAUTIN, R. (1984). Receptive fields and orientation shifts in the foveal striate cortex of the awake Macaque monkey. In G. Edelman, W. Gall, \& W. Cowan (Eds.), Dynamic Aspects of Neocortical Function (pp. 41-65). New York: Wiley.

GREENE, E. (1987). The relative contribution of contact and target lines in the magnitude of the Poggendorff effect. Perception, 16, 385-388. Greene, E. (1988). The corner Poggendorff. Perception, 17, 65-70.

GREEN, E., \& AL-QUADDOOMI, S. (I989). Use of segment arrays to evaluate the strength of angular iduction. Manuscript submitted for publication.

Weintraub, D. J., \& Brown, S. (1986). Perceptual plane geometry: Collinearity judgments probe the perceived orientation of an angle's sides. Journal of Experimental Psychology: Human Perception \& Performance, 12, 434-444.

(Manuscript received November 23, 1988.) 